\title{
Subdermal contraceptive implants
}

Emeka Oloto, FWACS, MRCOG, MFFP

Specialist Registrar in Obstetrics and Gynaecology, St. James's University Hospital, Beckett Street, Leeds LS9 7TF, UK. SCMO, Leeds Community and Mental Health NHS Trust, Leeds, UK

Lawrence Mascarenhas, MD, MRCOG, MFFP, M.Ed. (Cantab)

Consultant Obstetrician and Gynaecologist, Department of Obsterics and Gynaecology, C Floor, East Block, Queens Medical Centre, Nottingham NG7 2UH, UK

(Accepted April 22 2000)

\section{How to use a FACT}

A FACT is an up-to-date review of a subject relevant to the speciality, intended to help you fulfil your CPD requirements in your home or place of work. Whilst FACTs are edited and reviewed at various levels within the Faculty, the actual contents and views expressed are those of the authors and not the Faculty. More specifically, these reviews are not guidelines. The CEC is producing clinical guidelines separately.

FACTs have three sections: a review, a true/false test, and discussion points. To use a FACT to earn CPD credits you should do the following:

1. Working alone: Read the review and do the test. The answers are provided on page 183 so you can mark yourself. If there are points you are unsure about, disagree with, or need further clarification on, make a note of these for use at a later date. This should take you no more than 1 hour. Keep a record of having done this in your CPD diary and, unless indicated otherwise on the FACT, this will earn you 1 hour (DFFP), 1 credit (MFFP).

2. Working as a group: arrange a meeting of at least 1 hour with colleagues to discuss the discussion points given in the FACT (page 174) and any issues the participants have come up with as a result of reading the FACT. Keep a record of having done this in your CPD diary and, unless indicated otherwise on the FACT, this will earn you 1 hour (DFFP), 1 credit (MFFP).

\section{Introduction}

Subdermal contraceptive implants are an additional approach to meeting the world-wide need for more effective and acceptable birth control. They provide long-acting, highly effective and reversible contraception by achieving low and stable concentrations of synthetic progestogens and require little user compliance, thereby achieving user failure rates that are similar to the method failure rates. 1,2

\section{History and development}

Subdermal contraceptive implants may be divided into two main groups: non-biodegradable, some of which are now widely used, and biodegradable, which remain under development in clinical trials. These are summarised in Table 1, This review will focus on systems that are in use in the UK (Norplant ${ }^{\circledR}$ and Implanon ${ }^{\circledR}$ ) or nearing general availability (Norplant $\mathrm{II}^{\circledR}$ ). 
Table 1 Subdermal contraceptive implants currently in use or under development

\begin{tabular}{|c|c|c|c|c|}
\hline Implant & Number & $\begin{array}{l}\text { Progestogen } \\
\text { (per capsule or rod or pellet) }\end{array}$ & $\begin{array}{l}\text { Use } \\
\text { (years) }\end{array}$ & Phase of development \\
\hline \multicolumn{5}{|l|}{ Non-biodegradable: } \\
\hline Norplant $^{\circledR}$ & $6 \mathrm{C}$ & Levonorgestrel $36 \mathrm{mg}$ & 5 & Marketed* \\
\hline Norplant II ${ }^{\circledR}\left(\right.$ Jadelle $\left.^{\circledR}\right)$ & $2 \mathrm{R}$ & Levonorgestrel $70 \mathrm{mg}$ & 5 & Marketed \\
\hline Sino implant $\mathrm{I}^{\circledR}$ & $6 \mathrm{C}$ & Levonorgestrel $36 \mathrm{mg}$ & 5 & Marketed \\
\hline Sino implant II ${ }^{\circledR}$ & $2 \mathrm{R}$ & Levonorgestrel $75 \mathrm{mg}$ & $3-5$ & Marketed \\
\hline Implanon $^{\circledR}$ & $1 \mathrm{R}$ & Etonogestrel $68 \mathrm{mg}$ & 3 & Marketed** \\
\hline Nestorone (ST-1435) & $1 \mathrm{C}$ & Nor-progesterone $40 / 78 \mathrm{mg}$ & 2 & I-II \\
\hline Uniplant or Surplant & $1 \mathrm{R}$ & Nomegestrel acetate $38 \mathrm{mg}$ & 1 & I \\
\hline \multicolumn{5}{|l|}{ Biodegradable: } \\
\hline Capronor & $1 \mathrm{C}$ & Levonorgestrel (12 or $21.6 \mathrm{mg})$ & 1 & II \\
\hline Annuelle & $4 \mathrm{P}$ & Norethindrone $90 \%$; Cholesterol $10 \%$ & 1 & I \\
\hline
\end{tabular}

$\mathrm{C}=$ Capsule; $\mathrm{R}=\mathrm{Rod} ; \mathrm{P}=$ Pellet; $*=$ Marketed in the UK $* *=$ Currently marketed in the UK

generation subdermal contraceptive implant systems. They were developed in an attempt to reduce the problems of insertion and removal associated with the six-implant system $\left(\right.$ Norplant $\left.{ }^{\circledR}\right)$. Other reasons that motivated further development of implants included the need for them to be less obvious under the skin; the need for implants to degrade and not require removal at all; the need to use progestogens that are less androgenic than levonorgestrel so that acne, weight gain, and perhaps other side effects would be less common; and the need to use simple, disposable devices to speed insertion and reduce the risk of bloodborne disease transmission. ${ }^{4,5}$ Norplant II ${ }^{\circledR}$ received Federal Drug Agency (FDA) approval in 1996 and is marketed in Finland, while Implanon ${ }^{\circledR}$ has been approved by the European Drug Licensing Agency (EDLA) and is currently marketed in Australia, Indonesia and 11 European countries.

\section{Scientific basis for sustained-release contraceptives}

The major aims of non-oral routes of contraceptive steroids include long duration of action, continuous and steady steroid blood levels and avoidance of the 'first pass' peak effect through the hepatic portal system, thus avoiding the peak plasma levels of steroids associated with oral dosing without compromising contraceptive efficacy. ${ }^{1}$ The higher bioavailability leads to lower doses of steroids being administered, with a possible parallel reduction in the occurrence of adverse effects.

\section{Mechanism of action}

The mechanisms of action include: cervical mucus blockade and prevention of sperm penetration; ${ }^{6}$ suppression of oestradiol-induced cyclic maturation of the endometrial lining, causing hypotrophic changes $;{ }^{6}$ and ovarian effects ranging from disordered luteal phase through complete anovulation to persistent follicles. ${ }^{6}$ However, Implanon ${ }^{\circledR}$ was designed to achieve complete inhibition of ovulation during its period of action. ${ }^{7}$

\section{Advantages and disadvantages}

The efficacy of these methods is high: Norplant ${ }^{\circledR}$ has a cumulative failure rate of 1 per 100 users after 5 years of use $(0.26$ per 100 woman years $) ;{ }^{8}$ Norplant $\mathrm{II}^{\circledR}$ has a similar cumulative failure rate after 3 and 5 years of use of 0.8 per 100 users $^{9}$ and combined data from 13 studies with Implanon ${ }^{\circledR}$ indicated that no pregnancies occurred in 1716 women treated - 53530 cycles (4103 women years), resulting in a Pearl Index of 0.0 (95\% CI, $0.00-0.09){ }^{7}$ Based on the principle of informed choice and ability to discontinue method at any time for whatever reason, they require little user compliance or motivation, resulting in use-effectiveness rates which closely approximate theoretical effectiveness. In addition it may be used by women who have contraindications to oestrogens. ${ }^{2}$ Furthermore, fecundity returns immediately following removal. $^{2}$

On the other hand, insertion and removal of contraceptive implants are minor surgical procedures that require trained personnel and with risks similar to any minor surgical procedure (infection, bleeding or haematoma). Women cannot initiate or discontinue the method themselves, and may encounter difficulty in finding personnel trained in removal techniques. Finally, the disruption of menstrual pattern frequently associated with contraceptive implant use, and the visibility of the capsules in some cases, may be unacceptable to women or their partners. ${ }^{2,10}$

\section{Insertion and removal}

Contraceptive implants are inserted subdermally, superficially enough to be palpated. Users of nonbiodegradable implants must be able to have them removed whenever they want, for whatever reason, or at the end of their duration of contraceptive action, with minimal inconvenience or discomfort.

Insertion and removal of implants is performed under local anaesthesia, which is uncomplicated in the majority of cases. However, some problems associated with insertion and removal have been reported. ${ }^{11,12}$ Incorrect insertion makes removal much more difficult, which stresses the importance of training. ${ }^{2}$ Difficult Norplant ${ }^{\circledR}$ removals, using the standard technique as recommended by the manufacturers, have prompted some researchers to develop new methods for removal. ${ }^{13,14}$

The Faculty of Family Planning and Reproductive Health Care offers a training framework, which covers both the theoretical and practical aspects of subdermal contraceptive implant insertion and removal. Further details of this framework can be obtained from the Faculty.

Implanon ${ }^{\circledR}$ has several advantages over Norplant ${ }^{\circledR}$ with respect to insertion and removal: it is a single rod; the EVA carrier has a semi-rigid consistency facilitating removal, and finally it is conveniently preloaded within a sterile, disposable applicator. ${ }^{15}$ A meta-analysis of the data from seven prospective randomised studies in 1378 women showed that it was approximately four times quicker to insert and remove Implanon ${ }^{\circledR}$ as compared to Norplant ${ }^{\circledR}$ (mean insertion times 1. 1 Vs 4.3 minutes, respectively; 
mean removal times $2.65 \mathrm{Vs} 10.2$ minutes, respectively). ${ }^{15}$ Furthermore, Implanon ${ }^{\circledR}$ was associated with a significantly lower incidence of removal complications $(0.2 \% \mathrm{Vs} 4.8 \%$ respectively; $\mathrm{P}<0.001){ }^{15}$ Finally, the mean removal time for Norplant $\mathrm{II}^{\circledR}$ reported in another study was 4.9 minutes, i.e. twice that reported with Implanon ${ }^{\circledR}$ and half that required for Norplant ${ }^{\circledR} .16$

Implanon ${ }^{\circledR}$ is licensed for 3 years of continuous usage. The manufacturer's datasheet suggests that Implanon ${ }^{\circledR}$ may be less effective during the third year of use in women weighing more than $70 \mathrm{~kg}$. As there is no scientific evidence to support this, it is our normal practice not to treat women weighing more than $70 \mathrm{~kg}$ any differently from other women using this method.

\section{Side effects and management}

Subdermal contraceptive implants share common side effects, the most common of which is menstrual disruption $^{1,2}$ which is also the most common reason for discontinuation of the method and presents a major obstacle to its widespread use. ${ }^{17}$ Because the changes in menstrual bleeding pattern vary widely, it is not possible to predict the change a particular client may experience.

Over a period of 2 years, Implanon ${ }^{\circledR}$ produced a general picture of bleeding pattern incidence that was not dissimilar to that for Norplant ${ }^{\circledR}$, though amenorrhoea occurred more frequently, at about $30-40 \%$ compared with $20-30 \%$ for Norplant ${ }^{\circledR}$ between 12-24 months of use. ${ }^{18}$ Infrequent bleeding occurred in about $50 \%$ of women in the first 3 months, but declined to about $30 \%$ after 6 months. The incidence of prolonged bleeding was also higher in the first 3 months than subsequently, when it had an incidence of $10-20 \%$. Frequent bleeding was uncommon, usually less than $10 \%$ at any time over 2 years. However, it is important to remember that in untreated women of reproductive age, ${ }^{19}$ amenorrhoea occurred in about $1 \%$ of women aged about 30 years; infrequent bleeding in about $8 \%$, and frequent and prolonged bleeding in less than $0.1 \%$.

Other reported side effects such as weight changes, headaches, mood changes, abdominal bloating and discomfort, loss of libido, nausea, acne, alopecia etc. affect less than $10 \%$ of users. ${ }^{6}$ No significant difference has been reported in the side effect profile between Norplant ${ }^{\circledR}$ and Implanon ${ }^{\circledR}$.

The pathophysiological mechanisms of the menstrual disturbances are poorly understood. While there has been some progress in the understanding of the mechanisms of normal and abnormal menstruation, ${ }^{20}$ there is a relative lack of progress in the actual prevention and treatment of the menstrual disruption associated with progestogen-only contraceptives.

The only consensus of opinion appears to be thorough pre-treatment counselling about probable alterations to the menstrual cycle, and subsequent strong reassurance at follow-up visits. ${ }^{8,21}$ Inevitably, some patients will require specific treatment, but surveys of such treatments by experts around the World ${ }^{22}$ revealed absence of universal treatment criteria and variation in the type, dose and duration of use of medications such as combined oral contraceptives, oestrogens (synthetic or conjugated), progestogens (levonorgestrel, norethisterone, etc.), and non-steroidal anti-inflammatory drugs. Evidence-based management is not available for the other side effects associated with subdermal contraceptive implants.

An important positive message, however, is that continuation rates and user satisfaction levels are high among users who have undergone detailed pre-insertion counselling, in spite of the fact that these users also report a significant incidence of side effects, probably because the perceived advantages greatly outweigh the nuisance effects. $^{2,6,10}$ With good counselling, $83.4 \%$ and $53 \%$ continuation rates at 1 year $^{23}$ and 5 years, ${ }^{24}$ respectively, have been reported with Norplant ${ }^{\circledR}$. The 2-year continuation rate of Implanon ${ }^{\circledR}$ was reported to be $69 \% .{ }^{25}$

\section{The UK experience}

Norplant ${ }^{\circledR}$ was licensed in October 1993 and was the only subdermal contraceptive implant system in general use in the UK until September 1999. It is estimated to have been used by about 55000 UK women. ${ }^{26}$ World-wide, about six million women have used this implant, which is registered in 60 countries. ${ }^{8}$ The initial clinical experience with the method $^{23,27,28}$ was promising, although negative reports about Norplant ${ }^{\circledR}$ have appeared in the lay and medical press, ${ }^{29,30}$ mostly due to unrealistic method expectations and problems with removal. For economic reasons, the main distributor discontinued marketing Norplant ${ }^{\circledR}$ in the UK in November 1999, although it is still available from other sources.

The single-rod implant, Implanon ${ }^{\circledR}$, was licensed for use in the UK in September 1999. It is anticipated that this system should have significant advantages in terms of ease of insertion and removal.

\section{Conclusion}

In spite of the very low failure rates and high continuation rates, subdermal contraceptive implants have low usage within Europe. They have been found to be effective and acceptable methods of contraception in other countries. Detailed counselling about their advantages and disadvantages should precede insertion of the implants. Post-insertion counselling may also be necessary. Only trained professionals should insert and remove the implants. The main challenge for the future is to find appropriate therapy to alleviate bleeding problems, which are the main reason for the discontinuation of the method.

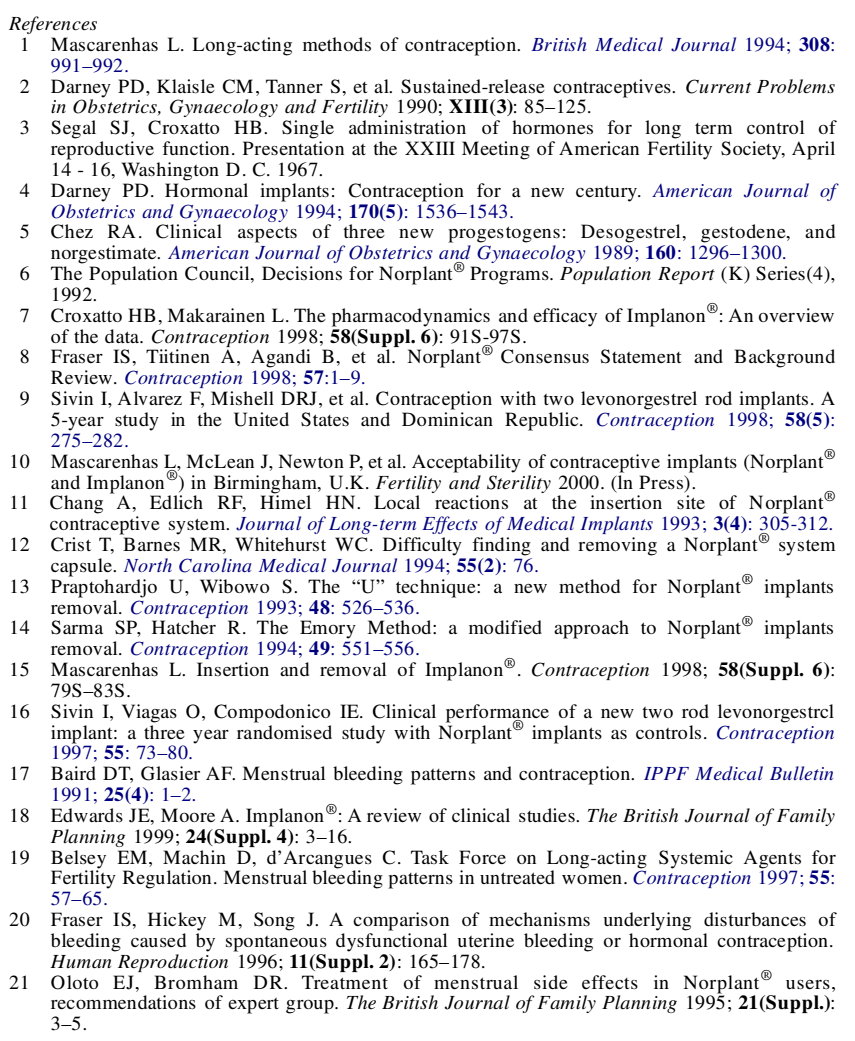


22 Nutley T, Dunson TR. Treatment of bleeding problems associated with progestin-only contraceptives: survey results. Advances in Contraception 1997; 13: 419-428.

Kirkman RJE, Bromham DR, O'Connor TP, et al. Prospective multicentre study comparing levonorgestrel implants with a combined contraceptive pill: final results. The British Journal of Family Planning 1999; 25: 36-40.

Sivin I, Campodonico I, Kiriwat 0, et al. The performance of levonorgestrel rod and Norplant ${ }^{\circledR}$ contraceptive implants: a 5 year randomised study. Human Reproduction 1998; 13(12): $3371-3378$.

Urbancsek J, Massai R, et al. A Multicentre efficacy and safety study of the single contraceptive implant Implanon ${ }^{\circledR}$. Implanon ${ }^{\circledR}$ Study Group. Human Reproduction 1999;

14(4): 976-981.

Eaton T. Hoechst Marion Roussel: The use of Norplant ${ }^{\circledR}$ in the UK. September 1998. Personal

Mascarenhas L, Lee S, Newton P, et al. First clinical experience with contraceptive implants Norplant and Implanon ${ }^{-}$) in the UK. British Journal of Obstetrics and Gynaecology 1996;

Peers T, Stevens JE, Graham J, et al. Norplant ${ }^{\circledR}$ implants in the UK: first year continuation and removals. Contraception 1996; 53(6): 345-351.

30 Roberts J. Women in US sue makers of Noirplant ${ }^{\circledR}$. British Medical Journal 1994; 309: 145.

\section{Discussion points}

1. Funding of training and service provision for contraceptive implants: whose responsibility is it?

2. Although the 'Norplant ${ }^{\circledR}$ Action Group' collapsed, what are the likely consequences on the future provison and utilisation of other contraceptive implants?

3. Management of menstrual disruption associated with the use of contraceptive implants.

4. The 'migrating implant': real or imagined phenomenon?

5. Should patients be informed at the end of the life span of the implants?

\section{F Faculty}

A Aid to

A CPD Self-Assessment Test

C CPD

T Topics

QUESTION SHEET

\section{Subdermal contraceptive implants}

Indicate your answer by ticking the appropriate box for each question

1. Norethisterone is the most widely used progestogen for contraceptive implants.

2. Counselling is the best treatment option for the menstrual disturbances associated with contraceptive implants.

3. The rate of release of steroids from contraceptive implants decreases proportionately to the surface area of the capsules, and increases with increasing wall thickness.

4. Norplant ${ }^{\circledR}$ is a six capsule implant system with each capsule containing $36 \mathrm{mg}$ of levonorgestrel.

5. Implanon ${ }^{\circledR}$ contains nomegestrol acetate, a second generation progestogen.

6. Mood swings are the most frequent side effect of subdermal contraceptive implants and the most common reason for discontinuation of the method.

7. It is easier to remove the implants than to insert them.

8. The two-rod contraceptive implant system is licensed for use in at least two countries.

9. Capronor, a biodegradable implant, is now in the phase II stage of development.

10. Implanon $^{\circledR}$ uses a semi-rigid EVA carrier.

\section{Turn to Page 183 for answers}

